\title{
Semiclassical interpretation of the spin interference effect observed in square loop arrays of $\mathrm{In}_{\mathbf{0 . 5 3}} \mathrm{Ga}_{\mathbf{0 . 4 7}} \mathrm{As} / \mathrm{In}_{\mathbf{0 . 5 2}} \mathrm{Al}_{\mathbf{0 . 4 8}} \mathrm{As}$ quantum wells
}

\author{
S. Mineshige, ${ }^{1}$ S. Kawabata, ${ }^{2}$ S. Faniel, ${ }^{1,3}$ J. Waugh,${ }^{1,4}$ Y. Sekine, ${ }^{5}$ and T. Koga ${ }^{1, *}$ \\ ${ }^{1}$ Graduate School of Information Science and Technology, Hokkaido University, Sapporo, Hokkaido 060-0814, Japan \\ ${ }^{2}$ Nanosystem Research Institute, National Institute of Advanced Industrial Science and Technology, Tsukuba 305-8568, Japan \\ ${ }^{3}$ ICTEAM Institute, Université catholique de Louvain, Louvain-la-Neuve, Belgium \\ ${ }^{4}$ Department of Physics, Virginia Polytechnic Institute and State University, Blacksburg 24061, USA \\ ${ }^{5}$ NTT Basic Research Laboratories, NTT Corporation, Atsugi, Kanagawa 243-0198, Japan
}

(Received 18 October 2011; revised manuscript received 4 November 2011; published 27 December 2011; publisher error corrected 30 December 2011)

\begin{abstract}
We present a semiclassical interpretation of the time-reversal spin interference (SI) observed in the square loop arrays made of $\operatorname{In}_{0.53} \mathrm{Ga}_{0.47}$ As quantum wells [T. Koga et al., Phys. Rev. B 74, 041302 (2006)]. The simulated amplitude of SI as a function of the Rashba parameter $\alpha$ captured characteristic features in the experimental results if $\gamma \lesssim 8 \mathrm{eV} \AA^{3}$ is assumed for the bulk Dresselhaus spin-orbit constant $\gamma$. Our work proves the validity of the semiclassical approach to predict the effect of time-reversal quantum interference in mesoscopic systems and the values of the spin-orbit coefficients recently deduced from the weak localization/antilocalization experiment.

DOI: 10.1103/PhysRevB.84.233305

PACS number(s): 85.35.Ds, 71.70.Ej, 73.23.Ad, 73.63.Hs
\end{abstract}

Introduction. The emerging field of spintronics ${ }^{1}$ has attracted much interest in the past decades. Among various aspects of spintronics, tremendous attention has been paid to the symmetry-breaking electric fields that lead to the gatecontrollable spin-orbit interactions (SOIs). ${ }^{2-4}$ The applications of these include the spin field-effect transistor, ${ }^{5}$ spin interference in mesoscopic rings ${ }^{6-10}$ or a qubit gate, ${ }^{11}$ and the nonmagnetic resonant tunneling spin filter. ${ }^{12}$ Recently, it is also suggested that these gate-controlled SOIs provide ingredients for the Majorana fermions to form topological qubits ${ }^{13}$ and induce the topological gaps in one-dimensional conductors. ${ }^{14}$ The SOIs, resulting from the bulk and structural inversion asymmetries, respectively, provide the linear/cubic Dresselhaus terms and the Rashba term in Hamiltonian, ${ }^{15-17}$ which are denoted as $H_{\mathrm{D}}^{(1)}, H_{\mathrm{D}}^{(3)}$, and $H_{\mathrm{R}}$, respectively. The explicit forms of these are $H_{\mathrm{D}}^{(1)}=\beta_{1}\left\{k_{y} \sigma_{y}-k_{x} \sigma_{x}\right\}, H_{\mathrm{D}}^{(3)}=\beta_{3}\left\{k_{\mathrm{F}} \cos (3 \chi) \sigma_{x}+\right.$ $\left.k_{\mathrm{F}} \sin (3 \chi) \sigma_{y}\right\}$, and $H_{\mathrm{R}}=\alpha\left\{k_{y} \sigma_{x}-k_{x} \sigma_{y}\right\}$, ${ }^{18}$ where $k_{\mathrm{F}}$ is the Fermi wave number, $\chi$ is the azimuthal angle for the in-plane wave vector $\mathbf{k}$, i.e., $k_{y} / k_{x}=\tan \chi, \sigma_{x}$ and $\sigma_{y}$ are the Pauli spin matrices, $\alpha, \beta_{1}$, and $\beta_{3}$ are the Rashba parameter and the linear and cubic Dresselhaus parameters, respectively. In the effective-mass approximation, $\alpha, \beta_{1}$, and $\beta_{3}$ are related to the more fundamental constants $a_{\text {SO }}$ and $\gamma$, the intrinsic Rashba and Dresselhaus spin-orbit constants, respectively, via $\alpha=$ $a_{\mathrm{SO}}\left\langle E_{z}\right\rangle, \beta_{1}=\gamma\left(\left\langle k_{z}^{2}\right\rangle-\frac{k_{\mathrm{F}}^{2}}{4}\right)$, and $\beta_{3}=-\frac{1}{4} \gamma k_{\mathrm{F}}^{2}$, where $\left\langle E_{z}\right\rangle$ is the averaged electric field within the quantum well (QW) derived from the confinement potential $V(z)$ and $\left\langle k_{z}^{2}\right\rangle$ is defined as $\left\langle\Psi(z)\left|-\left(\partial^{2} / \partial z^{2}\right)\right| \Psi(z)\right\rangle \cdot{ }^{19-22}$ While the simultaneous determination of $\alpha, \beta_{1}$, and $\beta_{3}$, equivalently the determination of $a_{\mathrm{SO}}$ and $\gamma$, has been a challenge even for a single material system, ${ }^{23,24}$ we recently succeeded in extracting these values for $\mathrm{In}_{0.52} \mathrm{Al}_{0.48} \mathrm{As} / \mathrm{In}_{0.53} \mathrm{Ga}_{0.47} \mathrm{As}(10 \mathrm{~nm}) / \mathrm{In}_{0.52} \mathrm{Al}_{0.48}$ As QWs from the weak localization/antilocalization (WAL) analysis of magnetoconductance (MC) data at dilution temperatures. ${ }^{21}$

In this Brief Report, we show that the semiclassical analysis of the spin interference (SI) effect ${ }^{9,25}$ observed in lithographically defined loop array structures also support these parameter values, which in turn support the applicability of the semiclassical schemes ${ }^{28-31}$ in the present problem. The SI effect, also termed as the time-reversal AharonovCasher effect, ${ }^{10,32}$ denotes the amplitude modulation of the ballistic analog $26,33,34$ of the Al'tshuler-Aronov-Spivak (AAS) oscillation $^{35,36}$ in the MC of the artificially defined loop array structures by the external gate voltage. We will test how well the SI effect observed experimentally ${ }^{9,25}$ can be described by the semiclassical consideration, where the channel width $(0.5 \mu \mathrm{m})$ of the loops is much larger than the Fermi wavelength $(\sim 25 \mathrm{~nm})$ so that the electrons retain the two-dimensional motions within the nanostructures. The reason why we arrayed the loops in these experiments was to average out quantum interferences other than the AAS effect. ${ }^{37}$ The advantage of square/rectangular shapes for the loops over a circle (or ring) shape is the possibility of easier separation of the anisotropic Dresselhaus terms from the isotropic Rashba term by controlling the aspect ratio of the loops and/or orienting the sides of the loops in some specific crystallographic axis. $8,25,38,39$ Despite the qualitative agreement between the experimental and theoretical results using an interferometer model, ${ }^{26}$ we also pondered on the model's quality, since the values of the $\mathrm{QW}$ thickness $d_{\mathrm{QW}}$ had to be varied typically by $\pm 10 \%$ from the designed value $(10 \mathrm{~nm})$ in order to fit the experimental data. ${ }^{38,39}$ In the present work, we will show that quantum interference considerations imposed on all classical trajectories of electrons within the designed nanostructures, obtained by the billiard simulations, clearly address these issues.

Simulation method. We performed ray tracing experiments (billiard simulation) for conduction electrons in twodimensionally extended nanostructures that are identical to those in experiment (see Fig. 1). An electron, injected from one of the leads (opened gaps) connecting neighboring loops, located on the left side of the unit loop structure in Fig. 1, undergoes a classical ray tracing motion. We varied the initial condition for the injected electrons $\left(\theta, Y_{0}\right)$ in the range $-90^{\circ}<\theta<90^{\circ}$ and $-150 \mathrm{~nm}<Y_{0}<150 \mathrm{~nm}$ (see Fig. 1 


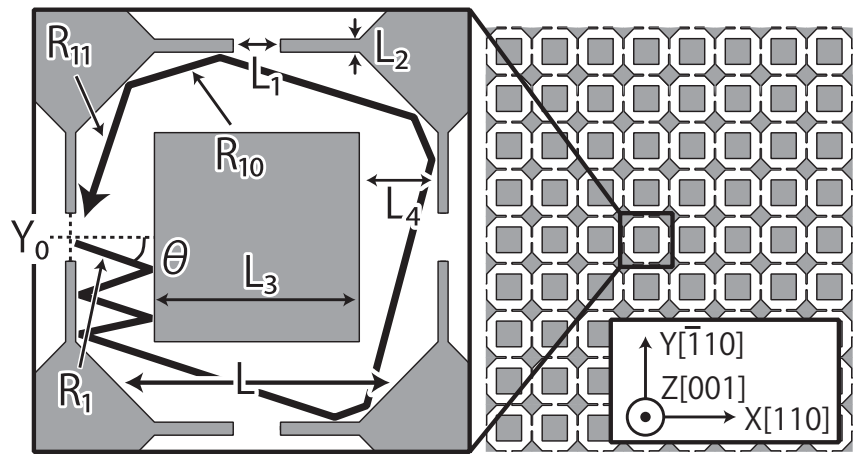

FIG. 1. Two-dimensionally extended square loop array structure used in the present simulation. The thick line with an arrow (left panel) represents an example of electron trajectories. $\mathbf{R}_{1}, \mathbf{R}_{10}$, and $\mathbf{R}_{11}$ denote the spin rotation operators for each segment of the trajectory indicated. The values for $L, L_{1}, L_{2}, L_{3}$, and $L_{4}$ are, respectively, 1.8, $0.3,0.1,1.3$, and $0.5 \mu \mathrm{m}$.

for the definitions of $\theta$ and $Y_{0}$ ). The ranges for $\theta$ and $Y_{0}$ were divided into 14399 and 59 discrete values with the steps of $0.0125^{\circ}$ and $5 \mathrm{~nm}$, respectively, yielding the total 849541 initial conditions. ${ }^{40}$ We allowed the particle to enter the neighboring loops sequentially throughout the extended two-dimensional (2D) network, although later it turned out that a similar simulation with an isolated square loop also reproduced the qualitative features we discuss in the present work. In each injection of electron, we stop the sequence of ray tracing when it reaches one of the following conditions: (1) the particle came back to the initial lead from the right, (2) the particle came back to the initial lead from the left, and (3) the cumulative length of the ray tracing experiment reached some maximum value $L_{\max }$, which was set to be 1 $\mathrm{mm}$ in the present study, a length longer than, but comparable to, the estimated phase coherence length $(0.5 \mathrm{~mm})$ in a typical experiment using a dilution refrigerator as discussed below. For the further interference consideration, we pick out only the trajectories belonging to (1) above (denoted as "closed trajectories"). This is because our experimental $-\Delta \sigma(B=0)$ vs $N_{\mathrm{S}}$ curves [see Ref. 9 or Figs. 2(b)-2(d)], where $N_{\mathrm{S}}$ is the sheet carrier density, are the manifestation of the selfinterference of electron wave function in the closed trajectories between the time-reversal directions. Here, we reiterate that $\Delta \sigma(B=0)$ is the amplitude of the FFT-filtered AAS primary oscillation $(h / 2 e$ period) in the electric conductivity, evaluated at $B=0$ relative to the mean value of $\sigma(B)$ for one oscillation period. ${ }^{41}$ We define the encircling area $S$ for each closed trajectory by $\frac{1}{2}|\oint \mathbf{r} \times d \mathbf{r}|$ where $\mathbf{r}$ is the position of the particle during the ray tracing experiment. We note that the magnetic flux piercing the closed trajectory is given by $B S$, which is associated with the Aharonov-Bohm phase. ${ }^{42}$ Since we focus our analysis on the primary frequency of the AAS oscillation $(h / 2 e$ period), we further screen the trajectories by the condition $0.5<S / S_{0}<1.5$, where $S_{0}=L^{2}, L$ being the side length of the ballistic square loop path as shown in Fig. $1(1.8 \mu \mathrm{m})$. We note that about $7.2 \%$ of the total injected electrons fell into this final category (61 478 out of 849541 ).

The SI amplitude of each closed trajectory is calculated applying the quantum-mechanical spin rotations $\mathbf{R}_{n}$

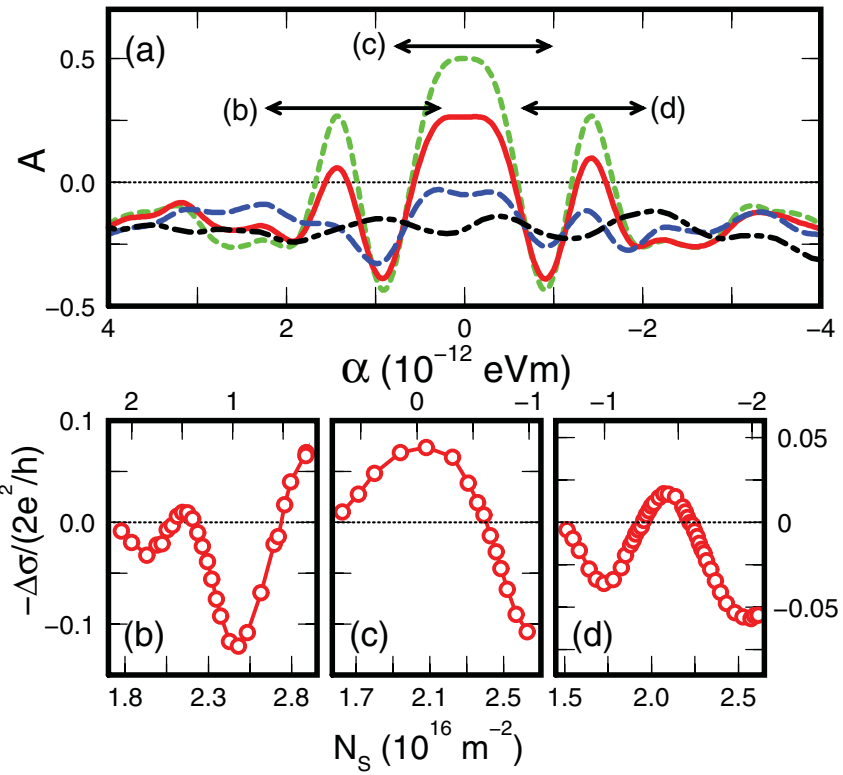

FIG. 2. (Color online) (a) Simulated spin interference (SI) patterns for the 2D-extended structure shown in Fig. 1. The dashed (green), solid (red), long-dashed (blue), and dash-dotted (black) curves denote the results with $\gamma=0,4,8$, and $27 \mathrm{eV} \AA^{3}$, respectively. The parts that reproduce the experiments shown in (b)-(d) are indicated by the horizontal bars with arrows at both ends. (b)-(d) Experimental SI patterns with $L=1.8 \mu \mathrm{m}$ (Ref. 9). The epiwafers used in (b)-(d) are, respectively, samples 2, 3, and 4 in Refs. 4 and 9. The ordinates for (b) and (c) are given on the left and that for (d) is given on the right side of the graphs. The approximate values of $\alpha$ in (b)-(d) are given on the top scale linearly correlating $\alpha$ with $N_{\mathrm{S}}$.

sequentially to an initial spin wave function $\Psi_{i}$. For example, the total spin rotation operator for the path shown in Fig. 1 is given as $\mathbf{R}_{\text {tot }} \equiv \mathbf{R}_{11} \mathbf{R}_{10} \cdots \mathbf{R}_{1}$. Then, the total spin rotation operator for the time-reversal direction of the same trajectory is given as $\left(\mathbf{R}_{\text {tot }}\right)^{-1}$. We note that $\mathbf{R}_{n} \equiv \exp \left(-\frac{i}{\hbar}\left\{H_{\mathrm{R}}+H_{\mathrm{D}}^{(1)}+H_{\mathrm{D}}^{(3)}\right\} t_{n}\right)$ where $t_{n}$ is the time it takes for an electron to travel through the $n$th segment of the given trajectory with length $\Delta l_{n}$, i.e., $t_{n} \equiv \Delta l_{n} / v_{\mathrm{F}}, v_{\mathrm{F}}$ being the Fermi velocity. Letting $\Psi_{\mathrm{F}}=e^{-2 \pi i\left(B S / \phi_{0}\right)} \mathbf{R}_{\mathrm{tot}} \Psi_{i}$ and $\Psi_{\mathrm{R}}=e^{2 \pi i\left(B S / \phi_{0}\right)}\left(\mathbf{R}_{\mathrm{tot}}\right)^{-1} \Psi_{i}$, where $\phi_{0}=\frac{h}{e}$, the intensity of the wave-function interference is given by $\frac{1}{4}\left|\Psi_{\mathrm{F}}+\Psi_{\mathrm{R}}\right|^{2}$, which is essentially the backscattering probability $P_{\text {bak }}$ in the ballistic SI model for an initial spin state $\Psi_{i} \cdot{ }^{26}$ After averaging $\frac{1}{4}\left|\Psi_{\mathrm{F}}+\Psi_{\mathrm{R}}\right|^{2}$ for the initial spin state $\Psi_{i}$ over the entire Bloch sphere, assuming that the incident electrons are spin unpolarized, $\frac{1}{4}\left|\Psi_{\mathrm{F}}+\Psi_{\mathrm{R}}\right|^{2}$ retains the form $\frac{1}{2}+A \cos \left(2 \pi \frac{B S}{\phi_{0} / 2}\right)$, where $A=\frac{1}{4} \operatorname{Tr}\left\{\mathbf{R}_{\text {tot }}^{2}\right\}$ after some algebra. $A$, denoted as the SI amplitude, is an oscillatory function of $\alpha, \beta_{1}$ and $\beta_{3}$.

Simulation results. Shown in Fig. 2(a) are the thuscalculated $A$ 's as a function of the Rashba parameter $\alpha$, averaged over all 61478 trajectories, for several selected values for $\gamma$. We note that we weighted $A$ of each trajectory with $\cos \theta$ to conserve the phase-space area represented in the Birkhoff coordinate ${ }^{28}$ in this calculation. We also note that the constant values $\left\langle k_{z}^{2}\right\rangle=5.8 \times 10^{16} \mathrm{~m}^{-2}$ and $k_{\mathrm{F}}=3.88 \times$ $10^{8} \mathrm{~m}^{-1}$ were used in the calculation. ${ }^{43} \mathrm{We}$ also tried 
exponentially decaying cutoffs in the ray tracing experiment, multiplying a factor $\exp \left(-L_{\text {tot }} / L_{\text {cutoff }}\right)$ to $A$ of each trajectory, where $L_{\text {tot }}$ is the total ballistic length of the given trajectory $L_{\text {tot }} \equiv \sum_{n} \Delta l_{n}$, to account for the possible spin and/or phase relaxations due to the structural imperfections and/or electron dephasing. However, it turned out that the introduction of $L_{\text {cutoff }}$ had only a minor effect if $L_{\text {cutoff }}>20 \mu \mathrm{m}$, since majority of the 61478 trajectories had ballistic lengths $L_{\text {tot }}$ smaller than $20 \mu \mathrm{m}$ (see Supplemental Material ${ }^{44}$ ). Hence all the data presented here were obtained without the exponentially decaying factor. In passing, the ballistic phase coherent length $L_{\phi} \equiv$ $v_{\mathrm{F}} \tau_{\phi}$ is estimated to be $0.5 \mathrm{~mm}$ in our samples using $\tau_{\phi}=5 \times$ $10^{-10} \mathrm{sec}$, which is obtained from the WAL analysis on nonpatterned samples at dilution temperature (electron temperature $\left.T_{e} \sim 100 \mathrm{mK}\right) .^{45,46}$ The same semiclassical simulation was also performed on a partially chaotic system, replacing the inner square island in the current structure with a circle island ${ }^{33}$ having the same area to test possible effects of irregular motions of electrons on the SI. However, the characteristic features in the SI patterns were almost unchanged. Further investigation on this aspect is currently underway.

It is intriguing to compare our simulated SI patterns in Fig. 2(a) with the experimental counterparts. Our previous experimental results were replotted in Figs. 2(b)-2(d). ${ }^{9} \mathrm{We}$ note that the characteristic features in simulation for $\gamma<$ $8 \mathrm{eV} \AA^{3}$ [see Fig. 2(a)], i.e., the main peak around $\alpha=0$, dips around $|\alpha|=1 \times 10^{-12} \mathrm{eV} \mathrm{m}$, and small peaks around $|\alpha|=1.4 \times 10^{-12} \mathrm{eV} \mathrm{m}$, were reproduced in experiment. We also note that the small peaks around $|\alpha|=1.4 \times 10^{-12} \mathrm{eV} \mathrm{m}$ are manifestations of the reentrance phenomena first predicted for regular quantum dots. ${ }^{30,31}$ The quantum simulations using the lattice Green-function method also reproduced the similar characteristic features in the SI pattern assuming $\gamma=0 .{ }^{27} \mathrm{De}-$ spite the excellent agreement between theory and experiment, some subtle issues have to be discussed before making our conclusions. (1) the $N_{\mathrm{S}}$ dependences of $\beta_{1}$ and $\beta_{3}$ were not taken into account in this simulation and (2) the suggested values for $\gamma\left(<8 \mathrm{eV} \AA^{3}\right)$ were considerably smaller than the $\mathbf{k} \cdot \mathbf{p}$ values for bulk $\mathrm{GaAs}$ and InAs $\left(\sim 25 \mathrm{eV} \AA^{3}\right) .{ }^{19}$ While the former reduces to a trivial issue if $\gamma<8 \mathrm{eV} \AA^{3}$, since the changes in the values of $\beta_{1}$ and $\beta_{3}$ as a function of $N_{\mathrm{S}}$ becomes negligible relative to that of $\alpha$, some comment may be required for the latter. There exists an extensive survey on the values of $\gamma$ for GaAs, showing the $\gamma$ values between 7.6 and $48 \mathrm{eV} \AA^{3},{ }^{47}$ where those in the lower end seem to be more plausible for $\mathrm{GaAs} / \mathrm{Al}_{1-x} \mathrm{Ga}_{x}$ As heterostructures. ${ }^{48}$ We also performed our semiclassical simulations using wide parameter ranges for $\gamma, \alpha$, and $N_{\mathrm{S}}$, even taking into account the $N_{\mathrm{S}}$ dependences in all the values of $\alpha, \beta_{1}$, and $\beta_{3}$, which revealed that $|\alpha|<1 \times 10^{-12} \mathrm{eV} \mathrm{m}^{-1}$ and $\gamma<8 \mathrm{eV} \AA^{3}$ are the only viable conditions for these parameter values in order to have large positive SI amplitude observed experimentally around $N_{\mathrm{S}} \approx 2.1 \times 10^{16} \mathrm{~m}^{-2}$ in Fig. 2(c).

In comparing the experimental SI patterns with the simulated ones, we note the direct relevance between $\left\langle E_{z}\right\rangle$ and $N_{\mathrm{S}}$, i.e., $\left\langle E_{z}\right\rangle=\frac{e}{\epsilon_{r} \epsilon_{0}} \frac{N_{\mathrm{S}}^{*}-N_{\mathrm{S}}}{2},{ }^{19}$ where $\epsilon_{r}$ is the relative dielectric constant $\left(\epsilon_{r}=13.1\right.$ for $\left.\operatorname{In}_{0.53} \mathrm{Ga}_{0.47} \mathrm{As}\right)$ and $N_{\mathrm{S}}^{*}$ is the sample dependent sheet carrier density at which $\left\langle E_{z}\right\rangle$ becomes zero. The values of $a_{\mathrm{SO}} m^{*}$ deduced from the WAL analysis were $a_{\mathrm{SO}} m^{*} / m_{e}=\left(1.46-1.51 \times 10^{-17} N_{\mathrm{S}}\left[\mathrm{m}^{-2}\right]\right) e \AA^{2},{ }^{21}$ where the
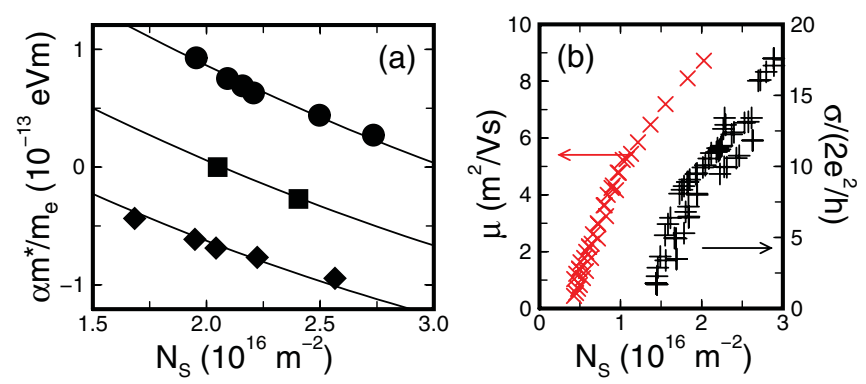

FIG. 3. (Color online) (a) The $\alpha m^{*}$ vs $N_{\mathrm{S}}$ relations obtained by correlating the peak, node, and dip positions in Figs. 2(b)-2(d) with those in the curve with $\gamma=4 \mathrm{eV} \AA^{3}$ in Fig. 2(a). The circle, square, and diamond symbols represent the values obtained from Figs. 2(b) 2(d), respectively. The solid curves are the fits discussed in the text. (b) The electric conductivities $\sigma$ of the nanofabricated samples $(+$ symbols) and the electron mobilities $\mu$ of nonpatterned epiwafers ( $\times$ symbols), as a function of the monitored sheet carrier density $N_{\mathrm{S}}$ using the nonpatterned region.

effective-mass value $m^{*} / m_{e}=0.047$ was used in the present simulation. In Figs. 2(b)-2(d), we can assign the values of $\alpha$ at the peak, dip, and node $(\Delta \sigma=0)$ positions in the experimental SI curves from the corresponding positions in one of the theoretical curves with $\gamma<8 \mathrm{eV} \AA^{3}$. Here, we plot the result using the theoretical curve with $\gamma=4 \mathrm{eV} \AA^{3}$ in Fig. 3(a) as a function of $N_{\mathrm{S}}$ together with the fits using the above relations for $a_{\mathrm{SO}} m^{*}$ and $\left\langle E_{z}\right\rangle$, where we use $N_{\mathrm{S}}^{*}$ as a fitting parameter. The values of $N_{\mathrm{S}}^{*}$ obtained from these fittings were $3.05,2.07$, and $1.24 \times 10^{16} \mathrm{~m}^{-2}$ for samples in Figs. 2(b)-2(d), respectively. A similar analysis on the $\alpha$ vs $N_{\mathrm{S}}$ relations for a bare Hall bar sample from the WAL measurements revealed $N_{\mathrm{S}}^{*}=2.2,1.24$, and $0.5 \times 10^{16} \mathrm{~m}^{-2}$ for the same respective epiwafers. The discrepancy in the values of $N_{\mathrm{S}}^{*}$ here between the nanofabricated samples and the bare Hall bars is probably due to the shift in the actual carrier density in the nanostructured region of the sample from that of the nonpatterned region even under the same gate voltage, where the values of $N_{\mathrm{S}}$ were always monitored in the nonpatterned region of the sample. ${ }^{9}$ In this respect, the conductivities $\sigma$ of the nanostructured samples and the mobilities $\mu$ of the nonpatterned samples were plotted as a function of the monitored $N_{\mathrm{S}}$ for all samples in Fig. 3(b). We find that the $N_{\mathrm{S}}$ value at which $\sigma$ vanishes is shifted to a lower value from that at which $\mu$ vanishes by about $1 \times 10^{16} \mathrm{~m}^{-2}$. We believe that this shift is also partially related to the observed shift in the value of $N_{\mathrm{S}}^{*}$ between the patterned and nonpatterned samples, while, more exactly, different mechanisms should be responsible for diminishing $\sigma$ and $\mu$, i.e., gate pinching-off of the constricted parts in the nanostructures for diminishing $\sigma$ and the gate depletion and increased scattering of the conduction carriers for diminishing $\mu$.

Conclusions. We performed semiclassical billiard simulations on nanofabricated square loop array structures made of $\mathrm{In}_{0.53} \mathrm{Ga}_{0.47} \mathrm{As}$ quantum wells to understand the observed time-reversal spin interference (SI) phenomena. We found that the necessary requirement for the value of the bulk Dresselhaus constant $\gamma$, for the simulated SI curves to agree 
with the experimental counterparts, is $\gamma<8 \mathrm{eV} \AA^{3}$, which is considerably smaller than the $\mathbf{k} \cdot \mathbf{p}$ value of $25 \mathrm{eV} \AA^{3}$. The peak and dip positions in the reentrant experimental SI curves were predicted very accurately by our semiclassical simulations using $a_{\mathrm{SO}} m^{*} / m_{e}=\left(1.46-1.51 \times 10^{-17} N_{\mathrm{S}}\left[\mathrm{m}^{-2}\right]\right) e \AA^{2}$ for the values of the intrinsic Rashba constant, the values deduced from the separate weak localization/antilocalization experiment. ${ }^{21}$ We conclude that our work addressed the powerfulness of the semiclassical approach to predict the mesoscopic phenomena, especially the time-reversal quantum corrections to the electric conductance in nanostructured devices.

Acknowledgments. The authors are indebted to K. Sueoka, T. Akazaki, R. Winkler, K. Saito, J. Nitta, and T. Ando for fruitful discussions. This work was partially supported by KAKENHI, Grant-in-Aid for Scientific Research (B), Grant No. 23360001, and Grants-in-Aid from Hokkaido Innovation through Nanotechnology Support (HINTS). S. Faniel thanks the Japan Society for the Promotion of Science (JSPS) for financial support. "koga@ist.hokudai.ac.jp

${ }^{1}$ J. Fabian, A. Matos-Abiaguea, C. Ertlera, P. Stano, and I. Zutic, Acta Phys. Slovaca 57, 565 (2007).

${ }^{2}$ J. Nitta, T. Akazaki, H. Takayanagi, and T. Enoki, Phys. Rev. Lett. 78, 1335 (1997).

${ }^{3}$ G. Engels, J. Lange, T. Schäpers, and H. Lüth, Phys. Rev. B 55, R1958 (1997).

${ }^{4}$ T. Koga, J. Nitta, T. Akazaki, and H. Takayanagi, Phys. Rev. Lett. 89, 046801 (2002).

${ }^{5}$ S. Datta, and B. Das, Appl. Phys. Lett. 56, 665 (1990).

${ }^{6}$ A. G. Aronov and Y. B. Lyanda-Geller, Phys. Rev. Lett. 70, 343 (1993).

${ }^{7}$ T. Z. Qian and Z. B. Su, Phys. Rev. Lett. 72, 2311 (1994).

${ }^{8}$ H. Mathur and A. D. Stone, Phys. Rev. Lett. 68, 2964 (1992).

${ }^{9}$ T. Koga, Y. Sekine, and J. Nitta, Phys. Rev. B 74, 041302 (2006).

${ }^{10}$ T. Bergsten, T. Kobayashi, Y. Sekine, and J. Nitta, Phys. Rev. Lett. 97, 196803 (2006).

${ }^{11}$ P. Foldi, B. Molnar, M. G. Benedict, and F. M. Peeters, Phys. Rev. B 71, 033309 (2005).

${ }^{12}$ T. Koga, J. Nitta, H. Takayanagi, and S. Datta, Phys. Rev. Lett. 88, 126601 (2002).

${ }^{13}$ S. Vishveshwara, Nat. Phys. 7, 450 (2011).

${ }^{14}$ C. H. L. Quay, T. L. Hughes, J. A. Sulpizio, L. N. Pfeiffer, K. W. Baldwin, K. W. West, D. Goldhaber-Gordon, and R. de Picciotto, Nat. Phys. 6, 336 (2010).

${ }^{15}$ G. Dresselhaus, Phys. Rev. 100, 580 (1955).

${ }^{16}$ E. I. Rashba, Fiz. Tverd. Tela (Leningrad) 2, 1224 (1960) [Sov. Phys. Solid State 2, 1109 (1960)].

${ }^{17}$ Yu. A. Bychkov and E. I. Rashba, Pis'ma Zh. Eksp. Teor. Fiz. 39, 66 (1984) [JETP Lett. 39, 78 (1984)].

${ }^{18}$ We note $\hat{x} \|$ [100], $\hat{y} \|$ [010] and $\hat{z} \|$ [001], where [111] is defined to be in the direction from group $\mathrm{V}$ to group III atoms in the present study. We also note that $\hat{z} \|[001]$ is defined to be normal to the epiwafer surface.

${ }^{19} \mathrm{R}$. Winkler, Spin-Orbit Coupling Effects in Two-Dimensional Electron and Hole Systems (Springer, Berlin Heidelberg, 2003).

${ }^{20}$ W. Knap, C. Skierbiszewski, A. Zduniak, E. Litwin-Staszewska, D. Bertho, F. Kobbi, J. L. Robert, G. E. Pikus, F. G. Pikus, S. V. Iordanskii, V. Mosser, K. Zekentes, and Y. B. Lyanda-Geller, Phys. Rev. B 53, 3912 (1996).

${ }^{21}$ S. Faniel, T. Matsuura, S. Mineshige, Y. Sekine, and T. Koga, Phys. Rev. B 83, 115309 (2011).

${ }^{22}|\Psi(z)\rangle$ is the confined wave function in the QW normal to the plane. We define $\left\langle E_{z}\right\rangle$ excluding the interfacial contributions in the present study (see Ref. 3).

${ }^{23}$ L. Meier, G. Salis, I. Shorubalko, E. Gini, S. Schon, and K. Ensslin, Nat. Phys. 3, 650 (2007)
${ }^{24}$ M. Scheid, M. Kohda, Y. Kunihashi, K. Richter, and J. Nitta, Phys. Rev. Lett. 101, 266401 (2008).

${ }^{25}$ T. Koga, H. Okutani, Y. Sekine, and J. Nitta, Phys. Status Solidi 3, 4220 (2006).

${ }^{26}$ T. Koga, J. Nitta, and M. van Veenhuizen, Phys. Rev. B 70, 161302 (2004).

${ }^{27}$ Z. Zhu, Q. F. Sun, B. Chen, and X. C. Xie, Phys. Rev. B 74, 085327 (2006).

${ }^{28}$ H. U. Baranger, D. P. DiVincenzo, R. A. Jalabert, and A. D. Stone, Phys. Rev. B 44, 10637 (1991).

${ }^{29}$ C. Chang, A. Mal'shukovb, and K. A. Chaoc, Phys. Lett. A 326, 436 (2004).

${ }^{30}$ O. Zaitsev, D. Frustaglia, and K. Richter, Phys. Rev. Lett. 94, 026809 (2005).

${ }^{31}$ O. Zaitsev, D. Frustaglia, and K. Richter, Phys. Rev. B 72, 155325 (2005).

${ }^{32}$ Z. Zhu, Y. Wang, K. Xia, X. C. Xie, and Z. Ma, Phys. Rev. B 76, 125311 (2007).

${ }^{33}$ S. Kawabata and K. Nakamura, J. Phys. Soc. Jpn. 65, 3708 (1996).

${ }^{34}$ S. Kawabata, Phys. Rev. B 58, 6704 (1998).

${ }^{35}$ B. L. Al'tshuler, A. G. Aronov, and B. Z. Spivak, Pis'ma Zh. Eksp. Teor. Fiz. 33, 101 (1981) [JETP Lett. 33, 94 (1981)].

${ }^{36}$ B. L. Al'tshuler, A. G. Aronov, B. Z. Spivak, D. Y. Sharvin, and Y. V. Sharvin, Pis'ma Zh. Eksp. Teor. Fiz. 35, 476 (1982) [JETP Lett. 35, 588 (1982)].

${ }^{37}$ C. P. Umbach, C. Van Haesendonck, R. B. Laibowitz, S. Washburn, and R. A. Webb, Phys. Rev. Lett. 56, 386 (1986).

${ }^{38}$ S. Faniel, T. Matsuura, S. Mineshige, Y. Sekine, and T. Koga, Physica E 42, 990 (2010).

${ }^{39}$ T. Koga, S. Faniel, S. Mineshige, T. Mastuura, and Y. Sekine, Phys. Procedia 3, 1325 (2010).

${ }^{40}$ These divisions for the initial conditions were confirmed to be sufficiently fine. For example, no noticeable changes were observed in the simulated results by reducing the number of divisions for $\theta$ by half.

${ }^{41}$ The fast Fourier transform (FFT) spectrum of the experimental $\sigma(B)$ was filtered with the frequency window $0.5 f_{0}<f<1.5 f_{0}$, where $f_{0}$ is the center of the AAS primary frequencies.

${ }^{42}$ H. U. Baranger, R. A. Jalabert, and A. D. Stone, Chaos 3, 665 (1993).

${ }^{43}$ The value of $\left\langle k_{z}^{2}\right\rangle$ was obtained by solving one-band Schrödinger equation and Poisson equation self-consistently for a 10-nm-thick nearly symmetric QW. The $k_{\mathrm{F}}$ value is from $N_{\mathrm{S}}=2.4 \times 10^{16} \mathrm{~m}^{-2}$.

${ }^{44}$ See Supplemental Material at http://link.aps.org/supplemental/ 10.1103/PhysRevB.84.233305 for the distribution of 61478 trajectrories with $L_{\text {tot }}$. 
${ }^{45}$ S. Faniel, T. Matsuura, S. Mineshige, Y. Sekine, and T. Koga (unpublished).

${ }^{46}$ The electron temperature in the experiments leading to Figs. 2(b)-2(d) seem to be higher than this $\left(T_{e} \gtrsim 1 \mathrm{~K}\right)$, where the ballistic phase coherent length $v_{\mathrm{F}} \tau_{\phi}$ is estimated to be $50 \mu \mathrm{m}$.
${ }^{47}$ J. J. Krich and B. I. Halperin, Phys. Rev. Lett. 98, 226802 (2007); See Supplemental Material at http://link.aps.org/supplemental/ 10.1103/PhysRevLett.98.226802.

${ }^{48}$ J. D. Koralek, C. P. Weber, J. Orenstein, B. A. Bernevig, S.-C. Zhang, S. Mack, and D. D. Awschalom, Nature (London) 458, 610 (2009). 